\title{
Morphological features of tumor in different treatment options for patients With Locally Advanced Breast Cancer
}

\author{
Narzieyva D.F ${ }^{1}$, Jonibekov J.J ${ }^{2}$., Erkinova Sh. $N^{3}$. \\ Bukhara state medical institute
}

\begin{abstract}
Despite significant advances in the early diagnosis and treatment of locally advanced breast cancer, 60-65\% of the Republic of Uzbekistan is accounted for by locally advanced breast cancer and its mortality rate is high. The morphological structure of breast cancer is important for the prognosis of the disease. On cell membranes there are special cytoplasmic proteins - receptors, which, when combined with hormones, can affect the rate of division of tumor cells. In terms of the choice of treatment options and the assessment of the forecast, some molecular genetic parameters of the tumor are important, among which, first of all, the hormonal status of the tumor (ER / PR), HER-2 / neu, p53, Bcl-2, Ki-67.
\end{abstract}

Key words: locally advanced breast cancer, morphological structure, hormonal status of a tumor.

\section{Introduction}

Breast cancer (BC) is still one of the main urgent problems of fundamental and clinical oncology, the intensity of which remains the highest among malignant neoplasms in women [1].According to a number of authors, in 2000, the average breast cancer incidence in Russia was $19.40 \%$, in Moldova $22.90 \%$, in Belarus $18.30 \%$, in Georgia $36.00 \%$, in Armenia $28.40 \%$, in Kazakhstan $18.30 \%$, in Uzbekistan $19.10 \%$ and is characterized by high neglect. (3)The incidence of breast cancer in women in Western countries is 5 times higher than in women living in Asian countries. In the western population, the incidence of breast cancer increases mainly in the postmenopausal age, while in Asian countries it is mainly in women under 50 years old, that is, at reproductive age.Despite significant advances in the early diagnosis and treatment of breast cancer, in the Republic of Uzbekistan 60-65\% is accounted for by locally advanced breast cancer and mortality from it is high and tends to constantly increase. The mortality rate of women with this localization of malignant tumor is in third place after diseases cardiovascular system and accidents, and in the first place in the structure of female mortality from cancer - 16.4\% (5) .In this case, the diagnosis is morphologically verified in $90-95 \%$ of cases.Stage I-II breast cancer is diagnosed in $60.3 \%$ of patients, stage III in $26.1 \%$ and stage IV in $12.4 \%$ of patients. The highest incidence rates are observed in the age groups 60 64.75 years and older : 134.6 and 137.68 per 100,000 population. (5)

\section{Main part}

In Uzbekistan, there is an increase in the number of primary breast cancer patients per 100,000 women, which amounted to 6.6 in the Republic of Uzbekistan as a whole. In the regions of Uzbekistan, the greatest increase in the incidence rate was noted in the Republic of Karakalpakstan - 1.7 times, and in Tashkent city - 1.5 times. Mortality from breast cancer also increased from 1985 to 2004, and this increase is greater in Bukhara and Surkhandarya. areas, 3.9 ; 3.8 times, respectively. [4]. The morphological structure of breast cancer is important for prognosis diseases. Invasive ductal carcinoma is most common up to $80 \%$. Special forms of invasive cancer, starting with lobular and lower, generally have a less malignant course than invasive ductal carcinoma, and therefore at one time were singled out into a special group.They make up about 15-20\% of all neoplasms. Tumors of mixed histological structure are quite common. In such cases, predominance of some type of cancer is noted.On cell membranes there are special cytoplasmic proteins - receptors, which, when combined with hormones, can affect the rate of division of tumor cells. Determination of the level of steroid hormone receptors (estrogens, progesterone, etc.) makes it possible to predict the course of the disease and to judge the sensitivity of the tumor to hormonal treatment.Locally advanced breast cancer differs from localized not only in the degree of tumor prevalence; in this variant, the frequency of tumors with a poor prognosis increases and the frequency of relatively favorable characteristics decreases.So, according to the Russian Oncology Center.N.N.Blokhin Russian Academy of Medical Sciences, the frequency of rare morphological forms of cancer with a relatively benign course (mucous, papillary, tubular and medullary) in early breast cancer (T1-2N0M0) is $11 \%$, and in MR BC - $2 \%$. Aneuploid tumors in early breast cancer were observed in $62 \%$, and in MR breast cancer - in $85 \%$, and the proportion of aneuploid-polyclonal neoplasms was $4 \%$ and 17\%, respectively [6]. 
According to some reports, in the case of the Infiltrative Form, the frequency of tumors with overexpression of HER2 neu increases to $40-50 \%$.

Among the huge number of molecular biological studies related to the prediction of breast cancer and the search for factors predicting the sensitivity of the tumor to various methods of treatment, one should include an integral assessment of a huge number of molecular biological characteristics of neoplasms, which is represented by the classification of breast cancer, based on variations in the set of expressed genes and the correlation of genetic characteristics of the tumor with long-term results. These characteristics also allow individualizing treatment and thereby improve its results [6,14]. In terms of choosing a treatment option and assessing prognosis, some molecular genetic parameters of the tumor are important, among which, first of all, the hormonal status of the tumor (ER / PR), HER-2 / neu, p53, Bcl-2, Ki-67 Surrogate isolation of the proposed BC subtypes has come into practice, based on the immunohistochemical study of ER, RP and HER2neu: the luminal type is characterized as ER + HER2neu-, HER2neu + subtype - any values of ER and RP with overexpression of HER2neu. basal subtype - RE-RP-HER2neu. Revision of the results of systemic therapy depending on the tumor subtype showed the predictive value of the new classification. Investigation of the ER / PR-status of breast cancer cells is performed by the immunohistochemical method in order to determine the indications for hormone therapy [17]. At the same time, immunohistochemistry helps in the choice of treatment for Neoadjuvant Poly Chemotherapy. Thus, with the neoadjuvant use of anthracyclines and taxanes in patients with breast cancer with ER (-), complete or partial morphological regression is more often recorded [13]. Interesting and conflicting results of studies on changes in the hormonal status of a tumor under the influence of chemotherapy.So LeeS.H.et al. [16] showed that the ER / PR status changes in $62 \%$ of cases after Neoadjuvant Poly Chemotherapy. On the contrary, Arens N. et al. [8] did not describe changes in ER / PR before and after treatment, while Kasami M. et al. [9] found changes only in the expression of progesterone receptors in the tumor under the influence of Neoadjuvant Poly Chemotherapy. It is known that epidermal growth factor, represented by the family of tyrosine kinase receptors, is involved in the regulation of breast cancer cell proliferation, among which the HER-2 / neuoncogene should be distinguished [10]. When HER-2 / neu is overexpressed, conditions are created for the activation of uncontrolled cell division processes, which is closely related to accelerated tumor growth and a tendency to invasive growth, early metastasis, high risk of recurrence, shortening of the overall and disease-free survival rates and indicates an aggressive course of the disease [12 ]. That is why current standards recommend evaluating HER-2 / neu, the level of which can be high both in the primary tumor and in lymphogenous metastases [11].

The most important indicator of the biological behavior of a malignant tumor is also the proliferative activity of its cellular elements. The size of the proliferative pool of tumor cells is assessed by the immunohistochemical method according to the proliferation marker, the K1-67 antigen. [2] It was found that a positive reaction to this antigen clearly correlates with the degree of tumor malignancy and has an inverse relationship with the level of estrogen and progesterone receptors, and is also a predictive factor for chemoradiation therapy [15].

Concluding, we can say that MR breast cancer is a systemic disease, the success of its treatment is associated with the progress of systemic therapy. The observed low efficiency of preoperative therapy (the presence of a residual tumor in $70-90 \%$ of cases) requires surgery and radiation therapy. An increase in the effectiveness of treatment in general has been proven when taxanes and capecitabine are included in the chemotherapy regimen, and trastuzumab for patients with HER2neu overexpression. [6,7] The molecular characteristics of the tumor increase the possibilities of individualization of treatment. In-depth study of the problem Locally advanced breast cancer makes it possible to expand the scientific understanding of the mechanism of carcinogenesis, to develop optimal approaches to improving the prevention, diagnosis and treatment of malignant neoplasms.

\section{References}

1. Bozhok AA, Semiglazov VF, Semiglazov VV Prognosis factors in breast cancer // Modern Oncology.-2005.-№1C.4-9.

2. Zaridze, D.G. Carcinogenesis / D.G. Zaridze. - M: Medicine, 2004 .-- 576 p.

3. Muratkhodzhaev N.K., Kosimov D.A. Breast cancer in Uzbekistan (statistical analysis) .- Tashkent, 2005.-P.428429.

4. Musabayeva A.I., Zhogina J.A., Slonimskaya E.M. The role of modern methods of radiation therapy in the complex treatment of patients with locally advanced forms of breast cancer // Materials of the I Congress of Oncologists of the Republic of Uzbekistan-Tashkent, 2005.-C 428 -429.5. Moiseenko, V.M. Modern drug treatment of locally spread and meta static burn of the mammary gland / V.M. Moiseenko, V.F. Semiglazov, S.A. Tyulandin. - SPb .: 
Grifon, 1997 .-- 254 p.

5. S.M. PORTNOY Russian Cancer Research Center named after N.N. blokhinARAMN", Moscow \|I TREATMENT OF LOCALLY ADVANCED BREAST CANCER-SIBERIAN ONCOLOGICAL JOURNAL. 2008. Appendix No. 2

6. Khorov A.O., K.N. Uglyanitsa, A.K. Grib - Journal of Grodno State Medical University No. 3 2012 -29-30 pages8 .Arens, N. HER2/neu, p53, Ki6 7, and hormone receptors do not change du ring neoadjuvant chemotherapy in breast ca ncer / N. Arens, U. Bleyl, R. Hildenbrand // Virchows Arch. -2005. - Vol. 446. - P. 48 9-49 6.

7. Comparison of estrogen receptor, progesterone receptor and Her-2 status in breast cancer pre- and post-neoadjuvant chemotherapy / M. Kasami [et al.] // Breast. - 2008. - Vol. 17. - P. 523-527.

8. Evaluation of the United States Food and Drug Administration- a pproved scoring a nd test system of H ER-2 protein expression in breast cancer / P. Birner [et al.] // Clin. Cancer Res. - 2001. - Vol. 7, № 6. - P. 1669-1 675.

9. Hamilton, A. The contribution of molecular markers to the prediction of response in the treatment of breast cancer: A review of the literature on HER-2, p53and BCL-2 / A. Hamilton, M. Piccart // Ann. Oncol. -2000. - Vol. 11, №6. - P. 647-663.

10. Hung, M.C. Basic science of HER-2/neu: a review / M.C. Hung, Y.K. Lau // Semin. Oncol. - 1999. - Vol. 26 , № 4. - P. 51-59. 14 .Joensuu, H. BcI-2 protein expression and long-term survival in breast cancer / H. Joensuu, L. PyIkkanen, S. Toikkanent // Am. J. Pathology - 1994. - Vol. 145, № 5. - P. 1191 - 1198.

11. Negative steroid receptors are a good predictor for response to preoperative chemotherapy in breast ca ncer (BC) results of a randomized trial / M. Untch [et al.] // Proc. Am. Soc. Oncol. - 2003. - Vol. 22 (abstract 35).

12. Pa letta, C.E. T he inferiorglu tea 1 free fla $\mathrm{p}$ in breast reconstruction / C.E. Paletta, J. Bostwick, F. Nahai // Plast. reconstr. Surg. - 1989. - Vol. 84. - P. 875.

13. Prognostic significance of Bcl-2 expression in stage III breast cancer patients who had received doxorubicin and cyclophosphamide followed by paclitaxel as adjuvant chemotherapy / K.H. Lee [et al.] / / BMC Cancer. - 2007. Vol. 7. - P.63.

14. Prognostic valu e of Ki-6 7 expression a fter short-term presurgica 1 endocrine therapy for prima rybreast ca ncer / W.J . Hrushesky [et al.] // J. Natl. Cancer Inst. - 2007. - Vol. 99, № 13. - P. 1053 . 25 .T he effect of neoadjuvantchemotherapy on estrogen and progesterone receptor expression a nd hormone receptor statu $\mathrm{s}$ in breast cancer / S.H. Lee [et al.] // Am. J Surg. - 2003. - Vol. 186. - P. 348 -3 50 .

15. Progestin a ction and progesterone receptor structu re in human breast cancer: a review / K.B. Horwitz [et al.] // Recent Prog. Horm.Res. — 1985. — Vol. 41. - P. 249 - 316. 